\title{
THE EFFICIENCY OF OLS ESTIMATORS OF STRUCTURAL PARAMETERS IN A SIMPLE LINEAR REGRESSION MODEL IN THE CALIBRATION OF THE AVERAGES SCHEME
}

Robert Kowal, Ph.D., Eng.

Kielce University of Technology

Faculty of Management and Computer Modelling

Al. Tysiąclecia Państwa Polskiego 7, 25-314 Kielce, Poland

e-mail: robertkk7@gmail.com

Received 13 July 2016, Accepted 16 November 2016

\begin{abstract}
A simple linear regression model is one of the pillars of classic econometrics. Multiple areas of research function within its scope. One of the many fundamental questions in the model concerns proving the efficiency of the most commonly used OLS estimators and examining their properties. In the literature of the subject one can find taking back to this scope and certain solutions in that regard. Methodically, they are borrowed from the multiple regression model or also from a boundary partial model. Not everything, however, is here complete and consistent. In the paper a completely new scheme is proposed, based on the implementation of the Cauchy-Schwarz inequality in the arrangement of the constraint aggregated from calibrated appropriately secondary constraints of unbiasedness which in a result of choice the appropriate calibrator for each variable directly leads to showing this property. A separate range-is a matter of choice of such a calibrator. These deliberations, on account of the volume and kinds of the calibration, were divided into a few parts. In the one the efficiency of OLS estimators is proven in a mixed scheme of the calibration by averages, that is preliminary, and in the most basic frames of the proposed methodology. In these frames the future outlines and general premises constituting the base of more distant generalizations are being created.
\end{abstract}

Keywords: simple linear regression model, OLS estimators, efficiency of OLS estimators, Cauchy-Schwarz inequality, conditional optimization 


\section{Introduction}

The surrounding economic reality is largely shaped by both economic phenomena and financial processes. For the majority of them identification, description or interpretation is a complex issue, frequently causing a multitude of problems. The reason for such complexity is a large number of interacting factors, often various in nature and character, multiplicity of layers and directions of their influence as well as other types of conditions or circumstances. That is why the models and methods used for their description and analysis ought to be, on the one hand, as simple as possible in numerical and interpretational terms, and on the other hand, provide possibly the most faithful reflection of reality in that regard. Therefore, unsurprisingly, linear models featuring the above listed characteristics in various shapes or forms constitute a fundamental tool for describing such types of phenomena and processes. Owing to their nature and character, a quantitative description of economic phenomena or financial processes must comprise two aspects: a statistical description and optimization. The underlining model for the description of such types of phenomena is a simple linear regression model. Despite the fact that it only links two factors, its role in various kinds of econometric studies is still relatively substantial. It is used, inter alia, in a sensitivity analysis, once the principle of ceteris paribus has been accounted for, an impact analysis and the force of influence that variables exert on one another, or in direct or indirect form it serves as a basic component of many different special-purpose models. Thus, recognizing its properties and nature in the widest and most varied aspects is an important issue, both from the theoretical and practical points of view. One of them involves the efficiency of OLS estimators of structural parameters, within the scope of which the first from variants of a completely new and methodogical approach is going to be presented being an important issue in this model. It will feature a simplicity and originality of solutions, cohesion of methodology, the use of enough simple tools, and dissimilarity from the outlines found in the literature.

\section{Simple linear regression model}

The model of a simple linear regression is defined as follows:

$$
y=\alpha+\beta x+\varepsilon .
$$

where:

$y$ - dependent variable,

$x$ - independent variable, 
$\alpha$ and $\beta-$ structural parameters,

$\varepsilon \quad-$ random component.

In the design of $n$-observations performed on $y$ and $x$ it is written as follows:

$$
y=\alpha+\beta x_{i}+\varepsilon_{i}, \quad i=1,2, \ldots, n .
$$

From a formal point of view, we have an additive composite of a random component with a simple linear dependency of variables. Such a design reflects the direct impact or influence of one factor and aggregation of all the other factors. It does not always entail direct economic reinforcement; nevertheless, it is highly convenient in numerical terms. The random component gives the model a stochastic character (with suitable assumptions) and it further determines its behaviour, since it collectively accumulates the influence of the variables omitted in the model. An adequate description of reality requires that suitable assumptions be taken in that regard. Typically, the so-called classic initial assumptions are made. The assumptions are as follows:

1. Invariance of an expected value. i.e. $E\left(\varepsilon_{i}\right)=0$ for all $i=1,2, \ldots, n$.

2. Variance stability $D^{2}\left(\varepsilon_{i}\right)=\sigma^{2}$ for all $i=1,2, \ldots, n$.

3. Independence between one another, i.e. $\varepsilon_{i}$ and $\varepsilon_{j}$ are independent for all $i$ and $j$.

4. Independence from $x_{i}: \varepsilon_{i}$ and $x_{j}$ are independent for all $i$ and $j$ (this is a natural assumption, since $x_{j}$ are non-random, i.e. $\varepsilon_{i}$ distribution does not depend on $x_{j}$ ).

5. Normality of distribution: $\varepsilon_{i}$ have normal distributions for all $i$ (a convenient assumption, though it may be weakened).

For the entire population the model equation assumes the following form:

$$
E\left(y_{i}\right)=\alpha+\beta x_{i}, \quad i=1,2, \ldots, n .
$$

It defines the function of regression in a population.

The ordinary least square (OLS) method is the most popular method for structural parameters estimation. The structural parameter estimators obtained in the model with this method are expressed in the following formulas:

$$
\left\{\begin{array}{l}
\hat{\alpha}=\bar{y}-\hat{\beta} \bar{x} \\
\hat{\beta}=\frac{\sum_{i=1}^{n}\left(x_{i}-\bar{x}\right)\left(y_{i}-\bar{y}\right)}{\sum_{i=1}^{n}\left(x_{i}-\bar{x}\right)^{2}}
\end{array}\right.
$$

The so-called OLS estimators are the functions of the following variables $y_{i}, x_{i}$, $i=1,2, \ldots, n$. 


\section{Efficiency of OLS estimators of structural parameters}

The efficiency of the estimator is important and usually a desired feature from the point of view of its practical applications. In the simple regression model efficient are estimators that are received with the least squares method. The matter of proving the efficiency of OLS estimators $\hat{\alpha}$ and $\hat{\beta}$ of the structural parameters of the simple regression model is separate and with one of the issues more placed in this model. The majority of literature on the subject points to, not without reason, to the multidimensional case (cf. e.g. Welfe, 2009). It is relatively simple to prove because it is directly based on deviations with uncomplicated apparatus. In the scheme of a uniform, general approach which we will present here being generally direct was not considered. One only knows a certain solution based on deviations and implemented directly from the multidimensional case (cf. Magnus, Katyszew, Pieriesieckij, 1997) as well as partial (cf. Maddala, 2006).

We will consider the matter of the OLS efficiency of estimators of structural parameters in the simple regression model:

$$
y_{i}=\alpha+\beta x_{i}+\varepsilon_{i}, \quad i=1,2, \ldots, n .
$$

In the framework of the standard system of deliberations of this issue which we took it is possible to present a few different creatively and characteristically (analytical, synthetic) variants of proving the efficiency of OLS estimators of structural parameters in a simple regression model.

The basis of the considered scheme will be aggregated with an appropriate constant additive calibration of averages, single or double, from secondary constraints i.e. conditions of unbiasedness of the estimator and if necessary identities constraint of the covariantional nature and implemented in the dependencies arrangement of it and the variance of this estimator being the Cauchy-Schwarz inequality. The idea of constructing such an aggregated from secondary constraints conditional constraint under the angle of estimation the value of the variance of the estimator of the structural parameter of the simple linear regression model with the help of Cauchy-Schwarz inequality is quite new and so far has never been implemented in this problem. This constraint alone, even in its basic form, allows in a simple manner to synthetize just enough or even in an optimal way in the scheme of interested us here estimation of the threshold value of variance of the estimator both implied in the unbiasedness of the estimators secondary constraints, which directly but separately in this manner one cannot implement in any case. 
Because of the different complexity of the process of proving we will start from a simpler case i.e. from proving the efficiency of the OLS estimator $\hat{\beta}$. Here we have:

$$
\hat{\beta}=\frac{\sum_{i=1}^{n}\left(x_{i}-\bar{x}\right)\left(y_{i}-\bar{y}\right)}{\sum_{i=1}^{n}\left(x_{i}-\bar{x}\right)^{2}}=\frac{\sum_{i=1}^{n} x_{i}\left(y_{i}-\bar{y}\right)}{\sum_{i=1}^{n}\left(x_{i}-\bar{x}\right)^{2}}=\frac{\sum_{i=1}^{n}\left(x_{i}-\bar{x}\right) y_{i}}{\sum_{i=1}^{n}\left(x_{i}-\bar{x}\right)^{2}}=\sum_{i=1}^{n} c_{i} y_{i}, D^{2}(\hat{\beta})=\sigma^{2} \sum_{i=1}^{n} c_{i}^{2}=\frac{\sigma^{2}}{\sum_{i=1}^{n}\left(x_{i}-\bar{x}\right)^{2}} .
$$

Let $\tilde{\beta}=\sum_{i=1}^{n} d_{i} y_{i}$ be any linear unbiased estimator of the structural parameter $\beta$. From the condition of unbiasedness:

$$
E(\tilde{\beta})=\sum_{i=1}^{n} E\left(d_{i} y_{i}\right)=\sum_{i=1}^{n} d_{i} E\left(y_{i}\right)=\sum_{i=1}^{n} d_{i}\left(\alpha+\beta x_{i}\right)=\alpha \sum_{i=1}^{n} d_{i}+\beta \sum_{i=1}^{n} d_{i} x_{i}=\beta,
$$

follow imposed on the weights secondary constraints:

$$
\left\{\begin{array}{l}
\sum_{i=1}^{n} d_{i}=0 \\
\sum_{i=1}^{n} d_{i} x_{i}=1
\end{array}\right.
$$

In this form these constraints are not suitable for direct implementation in the Cauchy-Schwarz inequality under the context of the efficiency of this OLS estimator. Bearing in mind, that the Cauchy-Schwarz inequality gives efficient estimation only in case of the one conditional constraint therefore in such a case like here before her implementing in the scheme of these secondary constraints and the variance of the estimator one must necessary initially perform the operation total of their transforming the type of the aggregation in one. Within this methodics we obtain them in the process of calibrating variables $x$ multiplying the first of them by $\bar{x}$ i.e. the additive calibrator of these variables i.e. the number calibrating these variables by which we multiply the proper equality and then deduct the second. As a result of this procedure we get the conditional aggregated constraint:

$$
\sum_{i=1}^{n} d_{i}\left(x_{i}-\bar{x}\right)=1
$$

The value of this calibrator was chosen on the principle of direct borrowing from deep conditioning fastened inside the simple regression model, mainly from formulas of variances of OLS estimators but peculiarly $\hat{\beta}$ and the same character of Cauchy-Schwarz inequality. 
Implementing in the scheme of this constraint as being conditional and the variance $\sigma^{2} \sum_{i=1}^{n} d_{i}^{2}$ of the estimator $\tilde{\beta}$ the Cauchy-Schwarz inequality we have:

$$
\sigma^{2} \sum_{i=1}^{n} d_{i}^{2} \sum_{i=1}^{n}\left(x_{i}-\bar{x}\right)^{2} \geq \sigma^{2}\left(\sum_{i=1}^{n} d_{i}\left(x_{i}-\bar{x}\right)\right)^{2},
$$

which gives here:

$$
\sigma^{2} \sum_{i=1}^{n} d_{i}^{2} \sum_{i=1}^{n}\left(x_{i}-\bar{x}\right)^{2} \geq \sigma^{2}
$$

hence:

$$
D^{2}(\tilde{\beta}) \geq \frac{\sigma^{2}}{\sum_{i=1}^{n}\left(x_{i}-\bar{x}\right)^{2}}=D^{2}(\hat{\beta})
$$

As we see in this case all of these are imposed by the proper scaling of this variance.

We will now prove the efficiency of OLS estimator $\hat{\alpha}$. This estimator has a slightly different character. On account of providing for the methodological cohesion both proves will be implemented above the methodology without changes at first. We have here:

$$
\hat{\alpha}=\sum_{i=1}^{n}\left(\frac{1}{n}-\frac{\bar{x}\left(x_{i}-\bar{x}\right)}{\sum_{i=1}^{n}\left(x_{i}-\bar{x}\right)^{2}}\right) y_{i}=\sum_{i=1}^{n} l_{i} y_{i}, D^{2}(\hat{\alpha})=\sigma^{2} \sum_{i=1}^{n} l_{i}^{2}=\sigma^{2}\left(\frac{1}{n}+\frac{\bar{x}^{2}}{\sum_{i=1}^{n}\left(x_{i}-\bar{x}\right)^{2}}\right) .
$$

Let $\tilde{\alpha}=\sum_{i=1}^{n} f_{i} y_{i}$ be any linear unbiased estimator of the structural parameter $\alpha$ From the condition of unbiasedness:

$$
E(\tilde{\alpha})=E\left(\sum_{i=1}^{n} f_{i} y_{i}\right)=\sum_{i=1}^{n} E\left(f_{i} y_{i}\right)=\sum_{i=1}^{n} f_{i} E\left(y_{i}\right)=\sum_{i=1}^{n} f_{i}\left(\alpha+\beta x_{i}\right)=\alpha \sum_{i=1}^{n} f_{i}+\beta \sum_{i=1}^{n} f_{i} x_{i}=\alpha,
$$

follow imposed on the weights secondary constraints:

$$
\left\{\begin{array}{l}
\sum_{i=1}^{n} f_{i}=1 \\
\sum_{i=1}^{n} f_{i} x_{i}=0
\end{array}\right.
$$

Multiplying first through the same as the earlier calibrator of variables and deducting the sides from second we obtain additively and the one time calibrated initial, here the conditional aggregated constraint: 


$$
\sum_{i=1}^{n} f_{i}\left(x_{i}-\bar{x}\right)=-\bar{x}
$$

Implementing in the scheme of this constraint as conditional and the variance $\sigma^{2} \sum_{i=1}^{n} f_{i}^{2}$ of the estimator $\tilde{\alpha}$ the Cauchy-Schwarz inequality we have:

$$
\sigma^{2} \sum_{i=1}^{n} f_{i}^{2} \sum_{i=1}^{n}\left(x_{i}-\bar{x}\right)^{2} \geq \sigma^{2}\left(\sum_{i=1}^{n} f_{i}\left(x_{i}-\bar{x}\right)\right)^{2},
$$

which gives here:

$$
\sigma^{2} \sum_{i=1}^{n} f_{i}^{2} \sum_{i=1}^{n}\left(x_{i}-\bar{x}\right)^{2} \geq \sigma^{2}(-\bar{x})
$$

and the result:

$$
D^{2}(\tilde{\alpha}) \geq \sigma^{2} \frac{\bar{x}^{2}}{\sum_{i=1}^{n}\left(x_{i}-\bar{x}\right)^{2}} .
$$

As one can see the received estimation is too weak to show the efficiency of this OLS estimator. The most probable reason of it seems to be the wrong calibration of variables $x$ or the initial form of the secondary constraints what could translate into the conditional aggregated constraint and directly in effect too weak threshold estimation of the variance of the estimator. The relatively rather small gap in the final estimation here is very regular $(1 / n)$ as well as, the calibration of $x$ variables here has being natural and deep authorisation inside the model impose to go towards the modification of this methodology and in trying to improve this threshold estimation and not search other qualitative approaches, without of course the function Lagrangean function. In this field we can only make the calibration of the weights of the distribution of this estimator. And indeed, by proper additional transformation this time the weights $f$ of the distribution of estimator $\tilde{\alpha}$ one can in a simple manner make this modification. The idea of the modification is to transform the weights $f$ of the distribution of estimator $\tilde{\alpha}$ in the manner: $g_{i}=f_{i}-\frac{1}{n}, i=1,2, \ldots, n$. It is worthwhile paying special attention to the identical here value of moving the weights of distribution of the $\tilde{\alpha}$ estimator and the gap received in the above estimation.

In the scheme of such calibration the weights of estimator $\tilde{\alpha}$ the secondary constraints have the form:

$$
\left\{\begin{array}{l}
\sum_{i=1}^{n} g_{i}=0 \\
\sum_{i=1}^{n} g_{i} x_{i}=-\bar{x}
\end{array} .\right.
$$


Multiplying the first of them by the same as the earlier calibrator $\bar{x}$ and deducting the sides from the second one we receive the following, equivalent that, aggregated constraint:

$$
\sum_{i=1}^{n} g_{i}\left(x_{i}-\bar{x}\right)=-\bar{x}
$$

Moreover it is like earlier and obtained in the same way, but now it is related to the moved weights $g_{i}, i=1,2, \ldots, n$ i.e. calibrated. Implementing by analogy as earlier the Cauchy-Schwarz inequality for this dependencies scheme we have:

$$
\sigma^{2} \sum_{i=1}^{n} g_{i}^{2} \sum_{i=1}^{n}\left(x_{i}-\bar{x}\right)^{2} \geq \sigma^{2}\left(\sum_{i=1}^{n} g_{i}\left(x_{i}-\bar{x}\right)\right)^{2}
$$

which gives here:

$$
\sigma^{2} \sum_{i=1}^{n} g_{i}^{2} \sum_{i=1}^{n}\left(x_{i}-\bar{x}\right)^{2} \geq \sigma^{2}(-\bar{x})^{2}
$$

hence we have an indirect estimation:

$$
\sigma^{2} \sum_{i=1}^{n} g_{i}^{2} \geq \sigma^{2} \frac{\bar{x}^{2}}{\sum_{i=1}^{n}\left(x_{i}-\bar{x}\right)^{2}}
$$

It is in addition the same kind of estimation which happened previously, but, the left-side is related to something completely different. Using the first of the secondary constraints we get the following dependence:

$$
\sum_{i=1}^{n} f_{i}^{2}=\sum_{i=1}^{n}\left(g_{i}+\frac{1}{n}\right)^{2}=\sum_{i=1}^{n}\left(g_{i}^{2}+\frac{2 g_{i}}{n}+\frac{1}{n^{2}}\right)=\sum_{i=1}^{n} g_{i}^{2}+\frac{2}{n} \sum_{i=1}^{n} g_{i}+\sum_{i=1}^{n} \frac{1}{n^{2}}=\sum_{i=1}^{n} g_{i}^{2}+\frac{1}{n} .
$$

Hence as a result we have:

$$
\sigma^{2} \sum_{i=1}^{n} f_{i}^{2} \geq \sigma^{2}\left(\frac{1}{n}+\frac{\bar{x}^{2}}{\sum_{i=1}^{n}\left(x_{i}-\bar{x}\right)^{2}}\right)
$$

The constant additive calibration of weights $f$ of the distribution of estimator $\tilde{\alpha}$ added to the earlier obtained threshold estimation of the variance still one, constant i.e. first component in this case. Thanks to that we have here by the way also the distribution of the variance $D^{2}(\hat{\alpha})$ of the OLS estimator $\hat{\alpha}$ to components with the full identification of sources. 
The obtained result is already satisfactory from a formal point of view of the targets. However, from the cognitive and interpretative side the feeling of being an unsatisfied researcher remains. The suggested here transformation of the variables, particularly weights $f$ of the distribution of the estimator $\tilde{\alpha}$ has a more combined rather than systematic character. Provided the calibration of variables $x$ do not raise some greater technical doubts, the calibration of weights $f$ of the distribution of the estimator $\tilde{\alpha}$ is being top-down forced and does not already have such a direct interpretation inside it. Due to these circumstances we will still present the outline which is definitely more general than the above scheme of inference. It is crucial to notice here, that initial here resulted aggregated constraint:

$$
\sum_{i=1}^{n} f_{i}\left(x_{i}-\bar{x}\right)=-\bar{x}
$$

is equivalent to equality:

$$
\sum_{i=1}^{n}\left(f_{i}-\mathrm{a}\right)\left(x_{i}-\bar{x}\right)=-\bar{x}
$$

where a - is any real number. From a formal point of view of these deliberations it is not only the parameter but the constant additive calibrator of the weights $f$ of the distribution of the estimator $\tilde{\alpha}$ and all at the same time the whole constraint. In just this constraint we have, unlike the previous case, two calibrators: one constant of variables $x$ the second "parametric variable" of weights $f$ of the distribution of the estimator.

This equality also results from the conditional secondary constraints of the estimator, where her receiving from initial their form is not already so direct as the straightforward aggregation used here so far and requires in dependence of the kind of approach their modification or supplements which, in other words, is being forced. It is possible to obtain it in the same manner as previously, where the secondary constraints must then have another form on entry:

$$
\left\{\begin{array}{l}
\sum_{i=1}^{n}\left(f_{i}-\mathrm{a}\right)=1-n \mathrm{a} \\
\sum_{i=1}^{n}\left(f_{i}-\mathrm{a}\right) x_{i}=-\mathrm{a} n \bar{x}
\end{array},\right.
$$

that is initially parametrically calibrated already with additive weights $f$ of the distribution of the estimator. It is equivalent to the initial one, but what is important here, is not aggregated directly from initial secondary constraints. It is also possibly different, if we want to preserve the unchanged form of the secondary constraints. In this case one needs to come from the extended of the known identity: $\sum_{i=1}^{n}\left(x_{i}-\bar{x}\right)=0$ for the arithmetic average set of equations: 


$$
\left\{\begin{array}{l}
\sum_{i=1}^{n} f_{i}=1 \\
\sum_{i=1}^{n} f_{i} x_{i}=0 \\
\sum_{i=1}^{n}\left(x_{i}-\bar{x}\right)=0
\end{array}\right.
$$

what of course unchangeable leaves also the feasible here area and to aggregate in the twostage procedure i.e. sequentially after two, how it took place so far, appropriately and additively calibrated constraints of appearing in turn. At first from the first two after the calibration of variables $x$ by average $\bar{x}$ we get the standard here aggregated constraint: $\sum_{i=1}^{n} f_{i}\left(x_{i}-\bar{x}\right)=-\bar{x}$ like earlier and then like the same from him and last constraint i.e. the identity after the calibration of weights $f$ of distribution of the estimator with parameter "a" already offer ultimate equality. And so this constraint is doubly additively calibrated: at first through constant $\bar{x}$ and then through the variable parameter "a" Let us make a note, what is important here, such a situation i.e. the direct or indirect way of obtaining the ultimate aggregated constraint from secondary constraints has transferring into the comparative aspect of different methodologies, in it particularly in the context of applying here for example the Lagrangean function and the solutions obtained with this method. Implementing in the scheme of it as a condition and additively calibrated variance by calibrator " $a$ " the Cauchy-Schwarz inequality we have:

$$
\sigma^{2} \sum_{i=1}^{n}\left(f_{i}-\mathrm{a}\right)^{2} \sum_{i=1}^{n}\left(x_{i}-\bar{x}\right)^{2} \geq \sigma^{2}\left(\sum_{i=1}^{n}\left(f_{i}-\mathrm{a}\right)\left(x_{i}-\bar{x}\right)\right)^{2} \text {, }
$$

which gives here:

$$
\sigma^{2} \sum_{i=1}^{n}\left(f_{i}-\mathrm{a}\right)^{2} \sum_{i=1}^{n}\left(x_{i}-\bar{x}\right)^{2} \geq \sigma^{2}(-\bar{x})^{2}
$$

and a further estimation of additively calibrated variance:

$$
\sigma^{2} \sum_{i=1}^{n}\left(f_{i}-\mathrm{a}\right)^{2} \geq \frac{\sigma^{2} \bar{x}^{2}}{\sum_{i=1}^{n}\left(x_{i}-\bar{x}\right)^{2}}
$$

The recalibration of her already gives the useful form a word of which is the estimation:

$$
\sigma^{2} \sum_{i=1}^{n}\left(f_{i}-\mathrm{a}\right)^{2}=\sigma^{2}\left(\sum_{i=1}^{n} f_{i}^{2}-2 \mathrm{a} \sum_{i=1}^{n} f_{i}+\sum_{i=1}^{n} \mathrm{a}^{2}\right)=\sigma^{2}\left(\sum_{i=1}^{n} f_{i}^{2}-2 \mathrm{a}+n \mathrm{a}^{2}\right) \geq \frac{\sigma^{2} \bar{x}^{2}}{\sum_{i=1}^{n}\left(x_{i}-\bar{x}\right)^{2}},
$$


from which we receive directly one parametric lower of the same variance of the estimator $\tilde{\alpha}$ :

$$
D^{2}(\tilde{\alpha}) \geq \frac{\sigma^{2} \bar{x}^{2}}{\sum_{i=1}^{n}\left(x_{i}-\bar{x}\right)^{2}}+\left(2 \mathrm{a}-n \mathrm{a}^{2}\right) \sigma^{2}=\sigma^{2} \mathrm{~F}_{1}(\mathrm{a}) .
$$

In order to get in the frames of this scheme of calibration the best of all possible here lower estimations of the variance of this estimator that is the threshold we must find the maximal value of the right side of the above inequality with regard to the parameter "a" The threshold function of the variance of the estimator $\tilde{\alpha}$ is here a quadratic function of the parameter "a" hence it is possible to use the simple scheme i.e. use directly a proper for this function formula of the threshold calibrator. Quadratic function $\mathrm{F}_{1}(\mathrm{a})$ of one variable:

$$
2 \mathrm{a}-n \mathrm{a}^{2}
$$

achieves the maximum value for:

$$
\mathrm{a}_{\max }=\frac{-2}{-2 n}=\frac{1}{n}
$$

and it is equal to $1 / n$, so we received, this time in the parametric variable scheme, the same result as previously. It is also obvious, that the above methodology, similarly as previously, which is a special case of her introducted into the threshold estimation of the variance obtained earlier as a result of copying the methodology of the verification of the efficiency of the OLS estimator $\hat{\beta}$ the same additional, in this case the first component, for need of its presence we recalled. Let us also still take note, what results from this scheme and what is closing the motif, past rather than closed until the end, that constant movement of the variance of the estimator $\tilde{\alpha}$ is possible from the right infinite range $\left\langle-\frac{1}{n}, \infty\right)$. This is a set of feasible values of the threshold here quadratic function: $F_{1}(a)=n \mathrm{a}^{2}-2 \mathrm{a}$.

The presented proof of efficiency of the OLS estimator $\hat{\alpha}$ in the whole comprehensive diameter though already very close, is not a full direct copy of the previous scheme until the end. Differences, at least perhaps already not typically qualitative, nevertheless are. Also from the cognitive side it is not truly complete, because both the same conception of the calibration of weights $f$ of the distribution of the estimator as well as the value of this calibrator further remain contextually loose inside the model i.e. inconsistent with the rest and in some ways isolated from the whole issue, even though the very choice was already made in a systematic manner. The cause of it is still unidentified and not-interpreted properly until the end the form of the conditional aggregated constraint, being the core of this methodology. Crucial in the context of explaining the cohesion and the uniformity it turns out here to be only not entirely 
a probable random observation, because however implied certain here with past conditioning and circumstances which right away we will see, resulting among others from the rushing here symmetry of variables pulling the suspicion also behind oneself the symmetry of calibrators. In this interpretation a received optimal value of the calibrator $1 / n$ is the average of weights $\bar{f}$ of the distribution of the estimator $\tilde{\alpha}$. The conditional aggregated constraint ultimate for the estimator $\tilde{\alpha}$ in this interpretative scheme already has a symmetrical relative to the variables appearing here and the weights and their calibrators form and what is important, typical:

$$
\sum_{i=1}^{n}\left(f_{i}-\bar{f}\right)\left(x_{i}-\bar{x}\right)=-\bar{x}
$$

Because it appears to the left side covariantional form that is fully taken back to the averages.

The above form of the conditional aggregated constraint already takes an effect uniform and cohesive methodically approach to proving the efficiency of both OLS estimators of the model of the simple linear regression in the arrangement of the form determined for them the Cauchy-Schwarz inequality.

In the arrangement of this constraint the following forms of the Cauchy-Schwarz inequality can be here implemented:

$\mathrm{I}: \quad \sigma^{2} \sum_{i=1}^{n}\left(f_{i}-\bar{f}\right)^{2} \sum_{i=1}^{n}\left(x_{i}-\bar{x}\right)^{2} \geq \sigma^{2}\left(\sum_{i=1}^{n}\left(f_{i}-\bar{f}\right)\left(x_{i}-\bar{x}\right)\right)^{2}$,

for the linear unbiased estimator of the structural parameter $\alpha$ and

II:

$$
\sigma^{2} \sum_{i=1}^{n}\left(d_{i}-\bar{d}\right)^{2} \sum_{i=1}^{n}\left(x_{i}-\bar{x}\right)^{2} \geq \sigma^{2}\left(\sum_{i=1}^{n}\left(d_{i}-\bar{d}\right)\left(x_{i}-\bar{x}\right)\right)^{2},
$$

for the linear unbiased estimator of the structural parameter $\beta$

or, which identical, in the equivalent shorter, with the right side like so far:

$$
\mathrm{I}: \quad \sigma^{2} \sum_{i=1}^{n}\left(f_{i}-\bar{f}\right)^{2} \sum_{i=1}^{n}\left(x_{i}-\bar{x}\right)^{2} \geq \sigma^{2}\left(\sum_{i=1}^{n} f_{i}\left(x_{i}-\bar{x}\right)\right)^{2},
$$

for the linear idle estimator of the structural parameter $\alpha$ and

$$
\text { II: } \quad \sigma^{2} \sum_{i=1}^{n}\left(d_{i}-\bar{d}\right)^{2} \sum_{i=1}^{n}\left(x_{i}-\bar{x}\right)^{2} \geq \sigma^{2}\left(\sum_{i=1}^{n} d_{i}\left(x_{i}-\bar{x}\right)\right)^{2},
$$

for the linear idle estimator of the structural parameter $\beta$.

The above forms of the Cauchy-Schwarz inequality are most general for every possible arrangement of the calibration of the variables $x$ and weights $f$ or $d$ of the distributions of both estimators with averages which as well are completely symmetrical. Well more here effective on 
account of the form and character of secondary constraints, particularly first. In the framework of the adopted above concept of the ultimate conditional aggregate constraint and answering it in the form of the Cauchy-Schwarz inequality in the target arrangement of the threshold estimation the value of the variance of the estimator from the low still referring to the fact is necessary why in the initial inequalities and in consequence in the conditional aggregated constraints or inversely just averages $\bar{x}, \bar{f}$ and $\bar{d}$ were applied, rather than other constant values acting as calibrators of present here variables and weights of distributions of the estimators doubly already calibrated additively the ultimate conditional aggregated constraint. In all of the considered cases we are dealing with quadratic forms of the same spherical type in a direct straight or "compound" i.e. multiplied form. It is known that the quadratic form $\sum_{i=1}^{n}\left(x_{i}-x\right)^{2}$ achieves the minimal value just for the arithmetic mean i.e. $\bar{x}$. In the initial here forms of the Cauchy-Schwarz inequality we have such just left-side " quadratic minimal" situation not always for very sums of squares $\sum_{i=1}^{n}\left(f_{i}-\mathrm{a}\right)^{2}$ and $\sum_{i=1}^{n}\left(d_{i}-\mathrm{b}\right)^{2}$ and $\sum_{i=1}^{n}\left(x_{i}-x\right)^{2}$ but, what is also in this separabel arrangement equivalent, in their products. The area of largest their sensitivities is because appropriate minimax descent together the minimum of the left hand with a maximum or a constancy right hand or inversely. Such inequality is so in the arrangement of choice about the averages as calibrators being the strongest, because:

$$
\min \sigma^{2} \Phi_{1}(\mathrm{a}, \mathrm{b})=\min \sigma^{2} \sum_{i=1}^{n}\left(f_{i}-\mathrm{a}\right) \sum_{i=1}^{n}\left(x_{i}-\mathrm{b}\right)^{2}=\sigma^{2} \Phi_{1}(\bar{f}, \bar{x}),
$$

for the estimator $\tilde{\alpha}$ and

$$
\min \sigma^{2} \Phi_{2}(\mathrm{a}, \mathrm{b})=\min \sigma^{2} \sum_{i=1}^{n}\left(d_{i}-\mathrm{a}\right)^{2} \sum_{i=1}^{n}\left(x_{i}-\mathrm{b}\right)^{2}=\sigma^{2} \Phi_{2}(\bar{d}, \bar{x}),
$$

for the estimator $\tilde{\beta}$ because determined in this way functions $\Phi_{1}(\mathrm{a}, \mathrm{b})$ and $\Phi_{2}(\mathrm{a}, \mathrm{b})$ achieve the smallest values exactly in points $(\bar{f}, \bar{x})$ and $(\bar{d}, \bar{x})$ appropriately.

Not only the considered above issue but also and other, easily noticeable, "are wiping" very close of power transferring to the effectiveness of the Cauchy-Schwarz inequality used here for the inference which is already directly being associated with optimization procedures. Due to the importance of, the already signalled as a matter of fact earlier, element of the approach it will be discussed individually and in the context most general from here possible: at first constant and then of the variable calibration of variables and weights of distributions but further alone only weights of the distributions of estimators. This motif is very closely related and already directly with optimization and has such a character. It is also in this problem the scope which is completely innovative and alone in itself constitutes a separate, sharply outlined cohesive whole. 


\section{Conclusions}

The proposed approach to prove the efficiency of OLS estimators based on the CauchySchwarz inequality and certain general properties, taking into account classical, often used associations seems to a large extent to simplify deliberations related to the simple linear regression model which brings also some new ideas to it. The simplicity of the inferences proofs combined with the ease and comprehensibility of the approach may predestine it to gain a place in the literature and the didactics of the subject. Simultaneously, it further proves that new approaches or solutions can still be found in models that, at present, are considered to be classic. Let us add still to the above suggested methodology that it has more distant and broader generalizations.

\section{References}

Dhrymes, P.J. (2000). Mathematics for Econometrics. Fourth Edition. New York: Springer.

Gujarati, D.N., Porter, D.C. (2009). Essentials of Econometrics. Fourth Edition. New York: McGraw-Hill.

Johnston, J. (1987). Econometric Methods. New York: McGraw-Hill.

Kmenta, J. (1986). Elements of Econometrics. New York: Macmillan.

Maddala, G.S. (2006). Ekonometria. Warszawa: Wydawnictwo Naukowe PWN.

Magnus, J.R., Katyszew, P.K., Pieriesieckij, A.A. (1997). Ekonometrika. Moskwa: Naczalnyj kurs, Delo.

Moss, C.B. (2015). Mathematical statistics for applied econometrics. CRC Press

Spanos, A. (1986). Statistical Foundations of Econometric Modelling. Cambridge: Cambridge University Press.

Stock, J.H., Watson, M.W. (2015). Introduction to Econometrics. PEARSON.

Weintraub, E.R. (1982). Mathematics for Economics. An Integrated Approach. Cambridge: Cambridge University Press.

Welfe, A. (2009). Ekonometria. Metody i zastosowania. Warszawa: Polskie Wydawnictwo Ekonomiczne.

Wooldridge, J.M. (2009). Introductory Econometrics. A Modern Approach. Fourth Edition, Canada: South-Western. 